\title{
Identification of matter flows in marginal filters of rivers (based on the principles of thermodynamics)
}

\author{
Aleksandr Khaustov $^{1 *}$, Margarita Redina ${ }^{1}$, and Inna Nemirovskaya ${ }^{2}$ \\ ${ }^{1}$ RUDN-University, Ecological Faculty, 117198 Miklukho-Maklaya st. 6, Moscow, Russia \\ ${ }^{2}$ Shirshov Institute of Oceanology of RAS, 117997, Nahimovskiy pr. 36, Moscow, Russia
}

\begin{abstract}
Material and energy flows in the marginal filters of rivers identified based on the analyses of the environmental fate of polycyclic aromatic hydrocarbons (PAH): Anthracene (An), Phenanthrene (Phen), Naphthalene (Naph), Pyrene (Py), Fluoranthene (Flu), Chrysene (Chr), Benzo[a]pyrene (BaP), Perylene (Pl). Experimental data on the components of the aquatic system of the marginal filter of the Northern Dvina River allow us to consider the processes of phase transitions of PAHs at geochemical barriers (GCB). Based on the principles of thermodynamics, a phenomenological model of migration and the formation of the most persistent PAH associations on the barriers is formed. The predominant possibility of $\mathrm{PAH}$ migration on suspended material, including biota, is shown. The probability of PAH accumulation between the most important components of aquatic systems was assessed according to the conditions of their migration activity in various zones of the marginal filter. Active and passive PAHs were determined in terms of their migration and accumulation in the components of aquatic systems. The role of PAHs as a reliable indicator of thermodynamic processes, including the development of technogenesis, is estimated.
\end{abstract}

\section{Introduction}

Challenges in the development of modern hydrology as science are largely due to the insufficient development of the methodology for constructing models linking quantitative parameters of water resources and the formation of their quality. Turning to the list of unsolved problems in hydrology [1], only one point is "responsible" for the qualitative assessments of hydrological objects. With the modern scale of technogenesis, this seems nonsense. Thus, discussing the development of hydrological science, we must pay more attention to the development of integrated models in hydrology reflecting not the quantity of water but also (sometimes in the first line) the qualitative characteristics.

Requirements for qualitative knowledge of hydrological processes put forward this issue to the fore. Despite the satisfactory quality of models of hydrological processes of runoff formation, they virtually cannot be associated with the processes of formation of the

\footnotetext{
* Corresponding author: khaustov-ap@,rudn.ru
} 
chemical composition of waters, even at a phenomenological level. There are only few well-known models based on numerical methods (brought to practical implementation), allowing to take into account the processes of turbulent diffusion and water regime. More or less justified there are also the methods of estimating permissible loads on water bodies taking into account water quality. Water bodies have several sources of nutrition, which are characterized by an individual chemical composition. This makes it possible to build complex models using the basic principles of thermodynamics.

Let us note that in the case of real (natural or man-made) systems, thermodynamic justifications are used only for theoretical constructions and generalizations. This is largely due to the lack of justified reliable markers (indication compounds) able to reflect adequately the complex processes of interaction of components of aquatic systems. This problem is extremely relevant when constructing run-off formation models. We propose to study these processes on the basis of the structural characteristics of the models identified in terms of thermodynamic parameters of flow geochemical markers.

\section{Research methods and objects}

In this article, to identify processes in aquatic systems, we analyze the transformation of $\mathrm{PAH}$ flows on GCB in the marginal filter system of the Northern Dvina river, a zone with a pronounced effect of technogenesis. GCB are considered as a system-forming potential for the formation of properties of aquatic systems. To analyze the energy of flows, models are created reflecting changing the thermodynamic parameters in aquatic systems (entropy, enthalpy, Gibbs energy of PAH associations migrating through GCB).

The data used present the PAH flows on 3 sampling stations: in the river zone, in the gravitational and physico-chemical zones of the marginal filter and in the sea zone [2]. The PAHs concentration coefficients $\mathrm{CC}$ were analyzed for the set of GCB in the studied aquatic system as the ratio of $\Sigma \mathrm{PAH}$ in the "receiving" medium to the $\Sigma \mathrm{PAH}$ in the releasing one [3]. We emphasize the role of dissolved organic matter in the formation of specific associations of PAHs. Most actively (with a change in concentrations of tens of thousands of times), these processes occur in a system with the participation of biota.

Among the considered media there are crio- and aerosols, lower and upper edges of ice, various layers of water, bottom sediments, biota (seston) and separation suspension [2]. The studies cover estimates of the activity of migration of PAH associations across the boundaries of the components of the aquatic system. From a hydrological point of view, such a methodology is close to estimates of the flows of matter in water bodies (Fig. 1).

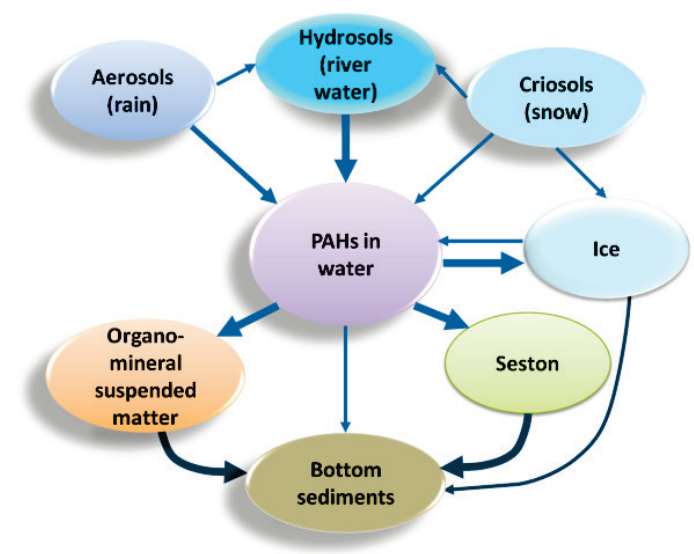

Fig. 1. Interaction of components in aquatic systems (bold arrows - dominant flows). 
From the point of view of geochemistry, GCB are the borders where transformation, accumulation, conservation and dissemination of substances and energy occur. Such borders can be divided in borders-barriers (like "water - ice", "water - bottom sediments" et al., in appliance with the classic view according to A.I. Perelman) and borders-partitions (like "water - water", "sediment - sediment") [4]. In the theory of systems, this division is fundamental, since it is possible to give various functions to extensive and intensive parameters, properties and behavior of components in creating migration flows of substances and energy. Borders are a necessary structural element of self-organizing systems. A variety of resonant forms of stable bonds in the system leads to the instability of borders. This paradoxical proposition forces a fundamental review of the functions of GCB. Borders can predetermine the nonlinearity of many processes. Thus, the evolution of systems can occur from boundary regions or their boundary states [5]. Of greatest research interest are the interfaces of media in various states of aggregation. It is here that selective maximization of mass transfer occurs due to the gradient of physicochemical parameters at the phase separation. The phase is understood as the physically homogeneous element of the system, limited by the interface, along which it (at least conditionally) can be separated from others. Of great interest is the zone of interaction of river and sea waters, where the barriers-borders "river water - sea water - bottom sediments" are presented. Such an interaction is most successfully interpreted within the concept of "marginal filter" [6].

To analyze the energy of the interaction of flows in aquatic systems, models are created for changing thermodynamic parameters (entropy, enthalpy, Gibbs energy of PAH associations migrating through GCB). The studies cover the estimates of the activity of migration of PAH associations across the boundaries of the components of the aquatic system. From a hydrological point of view, such a methodology is close to estimates of the flows of matter in water bodies (Fig. 1).

For the considered objects, the energetics of accumulation of marker compounds is most pronounced - from the minimum levels in atmospheric aerosols (with obvious differences for background and technogenic pressured control points) to the maximum values in bottom sediments and biota. For the first time, the role of living matter as an active component in the conversion of energy and mass flows of the aquatic system is quantified.

The research methodology is based on the ability of hydrocarbons to the natural decomposition in various phase states of aquatic systems. The material composition of hydrocarbons largely determines the degree of degradation. The more complex the atomicmolecular structures of hydrocarbon compounds, the less intense are the processes of their natural decomposition. Because of the flat form of PAH molecules due to the aromatic structure, PAHs are lipophilic; they tend to adsorb to many surfaces. Low solubility of PAHs explains the lack of high concentrations in fresh waters. The traditional representatives of PAHs in water are Naph, Flu, Phen, Py, and An. They are more soluble than heavy PAHs having 5-8 benzene rings. Consequently, light PAHs are a good indicator of many processes if they accumulate in water. Industrial pollution indicators are Py, Flu, 1,12-benzperylene, 3,4-benzofluorantene and 2,3-o-phenylenepyrene. However, all these hydrocarbons are extremely rarely analyzed in the environment and, especially, in aquatic organisms ; they can act as environmental risk factors. Depending on the genesis, PAHs are differentiated: $\mathrm{Py}$ and $\mathrm{BaP}$ are typical for objects of pyrogenic nature; naftigenesis is characterized by the accumulation and transformation of phenanthrenes; phenanthrenes, chrysens and perylene are representative of biogenesis $[7,8]$.

\section{Results and discussion}

The relationships between the components of the aquatic system in the form of PAH flows are presented (Fig. 2). The overall "rating" of PAH concentration activity at the borders can 
be represented as the following series: "seston - water"> "separation suspension - water"> "bottom sediments, contaminated - water"> "bottom sediments, relatively clean - water"> "upper ice edge - ice bottom edge (sea conditions)", etc.

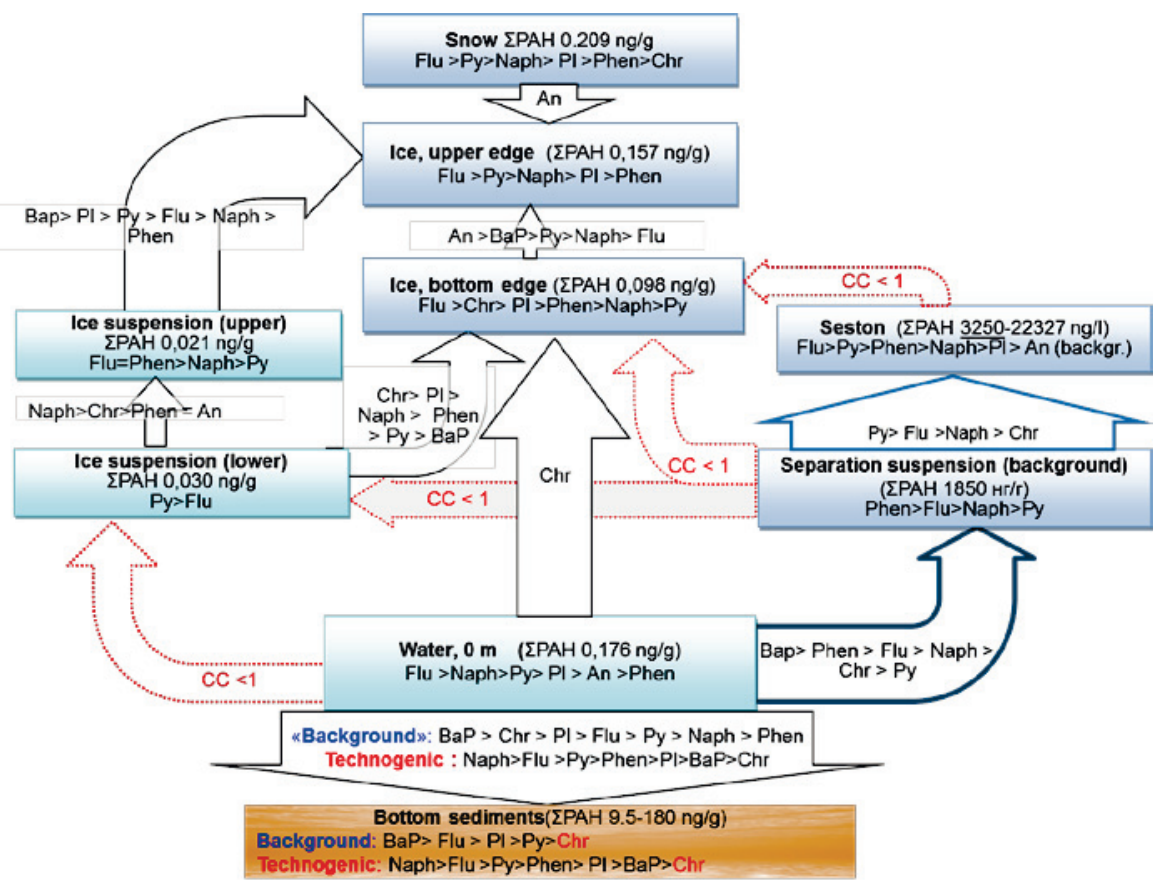

Fig. 2. PAH flows in the aquatic system of the Northern Dvina river, taking into account their genesis and fractionation under phase transitions

The energy carrier is fluctuating material particles, the sizes of which and the physicochemical state can be different and depend on "a variety of factors." On this postulate, in fact, the thermodynamics of nonequilibrium processes is based. The main thing is the irreversibility of these processes in natural systems. According to I. Prigogine, the properties of almost all dissipative open systems depend on time and evolve towards a decrease in the production of entropy. It is accompanied by the accumulation of mass of matter as a result of the manifestation of energy processes with negative entropy. However, the expenditure of free energy (and, hence, matter) for the formation of a number of processes is very individual and does not always obey I. Prigogine's theorem on minimizing of energy in nonequilibrium systems during the transition from one medium to another.

When working with empirical material, for natural environments the transitions of associations of chemical elements and compounds are revealed, which are very far from the theoretical concepts of classical thermodynamics about minimizing energy [3, 5]. Obviously, fractionation of PAHs will most contrastly occur during transitions between interconnected system components. The variability of the parameters of geochemical systems (evolution or orientation) should be adequately identified by the parameters of the production (increment) of entropy. At a zero value of this parameter, the interactions of its components are stationary or close to this. From these positions, it is proposed to identify the interaction of the flows of substances in the components of geochemical systems based on the construction of relationships between the increment of entropy and concentration of substances (Fig. 3). 
The extreme values on the graph correspond to the maximum negative $\Delta \mathrm{S}$ values - the accumulation of technogenic PAHs in the bottom sediments of the port Ekonomiya due to their transition from seston. At this HCB "separation suspension - bottom sediment", the polyarene lipophilic property is clearly manifested. As a result, stable associations of polyarenes were formed in the most saturated PAH deposition medium.

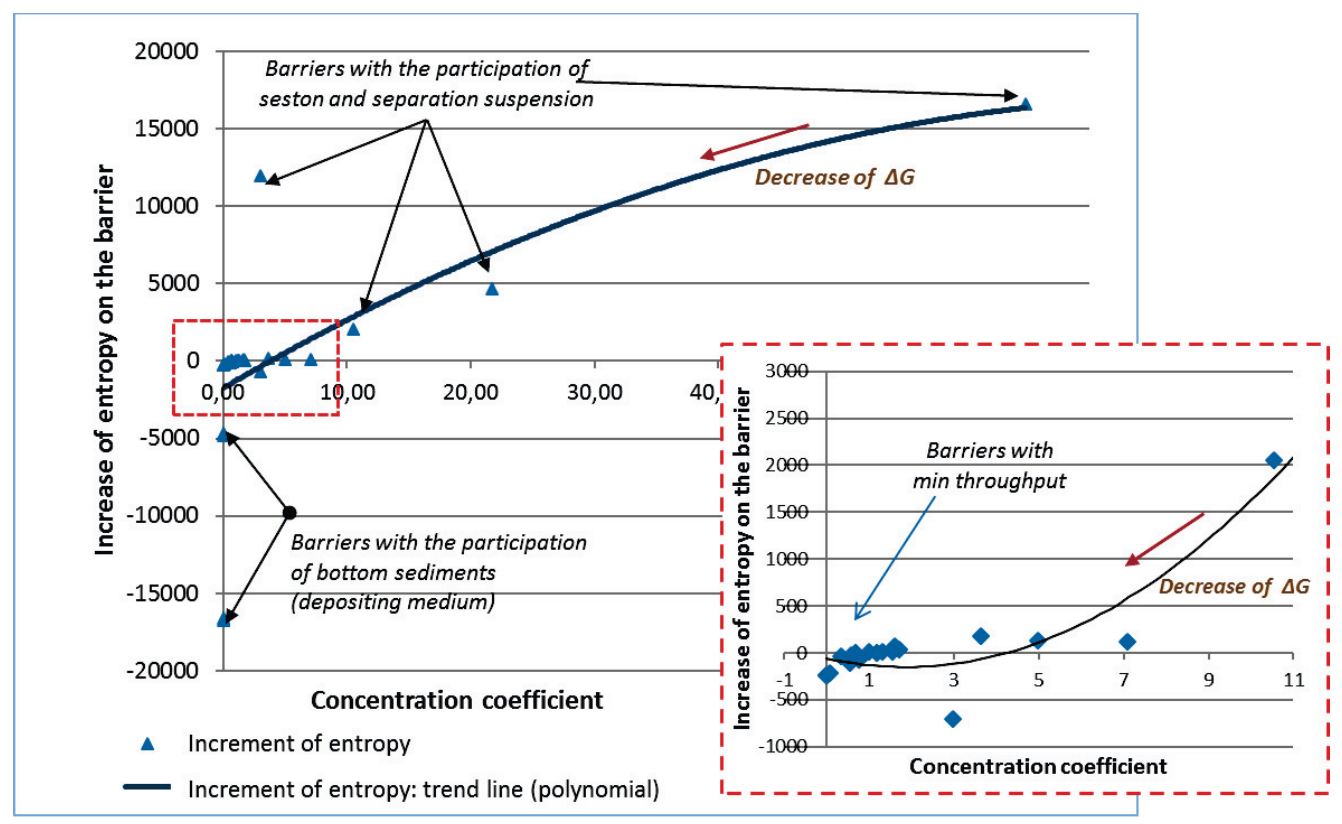

Fig. 3. The dependence of the entropy increment on the concentration of PAHs at contacts of the media

Maximum positive values: transition during the border "water - seston"; "water (layer $14 \mathrm{~m}$ ) - bottom sediments in the area of Mudyug island"; "seston - separation suspension", "water $0 \mathrm{~m}$ - separation suspension". Unstable flows of PAHs are recorded here, due to both the atomic-molecular properties of the PAHs and the properties of the receiving media with the active participation of living matter and suspensions, which contribute to the activation of sticking of active PAHs. As can be seen in Fig. 3, a "stable" picture of PAH flows is characteristic of the GCB system without the participation of living matter. Stability lies in the fact that the media exchange stable flows of matter and energy with each other, with the tendency of free energy to zero values. This fundamentally changes with the introduction of carbohydrate-based object flows (GCB) into the model.

Ice cover (from the standpoint of synergetics) is a metamorphosis of the active medium, capable of spatiotemporal self-organization, which in the process of homophase evolution can take fuzzy forms of boundaries due to the nonequilibrium of the interacting processes. This is expressed in the transition from spatial boundaries to the time limits of the matter and energy flows. Therefore, GCBs are clearly identified by a change in the dominants of PAHs isomers, not only during phase transitions at the partition boundaries, but also at the barrier boundaries. This thesis is clearly illustrated by the migration series on GCB.

The flow of PAHs into bottom sediments is significantly different from their combination in dissolved form, which indicates the potential ability of fractionation on suspensions of organogenic and mechanical composition. Technogenic PAHs are more actively converted to bottom sediments under favorable hydrodynamic and physicochemical conditions with subsequent accumulation. 


\section{Conclusion}

Estimates of the activity of migration of PAH associations through GCB in the aquatic system are obtained. For the considered objects, the energetics of accumulation of marker compounds are most pronounced - from the minimum levels in atmospheric aerosols (with obvious differences for background and technologically loaded control points) to maximum values for bottom sediments and biota. For the first time, the role of living matter as an active component in the energy of the aquatic system is quantified.

The increase in entropy acts as a characteristic of the functional diversity of the interaction of media on GCB with a simultaneous increase in the processes of concentration of substances in the "receiving" media. Such an approach to modeling can be used to highlight the evolutionary processes of subsystems (components) of the aquatic system. The advantage of this approach is the generalization of seemingly heterogeneous processes in the system into hierarchical subordination.

These conclusions are confirmed by calculations of thermodynamic characteristics and empirical relationships between them for various associations of PAHs, their sum and concentration coefficients CC. The latter characteristic can be considered as the most informative parameter of the effectiveness of the nonequilibrium macro-system in the framework of "introduction - transformation (or transformation) - removal of substances".

Our calculations link the decrease of the Gibbs free energy with an increase of CC for the barriers formed by the "non-living" components (without the participation of biota).

Barriers or media contacts are selective geochemical "filters" for PAHs, which quite clearly reflect the transition of a macro system from a kinetic state to a thermodynamic one.

From the standpoint of the macrokinetics of the phase transformations of matter in nonequilibrium systems, such contacts reduce the value of thermodynamic forces that facilitate the migration of substances. At the same time, they form breakthrough flows, causing self-oscillations of the giving and receiving systems.

The material has been prepared in the framework of the 5-100 project, financially supported by the Ministry of Education and Science of the Russian Federation (the Agreement № 02.A03.21.0008). Presented models are built on the base of data obtained in the RFBR projects 17-05-00356, 18-0580049 and modeling approaches developed in the RFBR project 07-05-07046.

\section{References}

1. G. Blöschl, M. F. Bierkens, A. Chambel, C. Cudennec et al., Hydrol. Sc. J., 64(10), 1141-1158 (2019).

2. I. A. Nemirovskaya, Oil in the ocean. Pollution and natural flows (Moscow, Nauchny mir, 2013) (in Rus.).

3. A. P. Khaustov, RUDN J. of Ecol. and Life Safety, 25(3), 396-413 (in Rus.).

4. A. Yu. Opekunov, Environmental sedimentology (Saint-Petersburg Univ. Publ., Saint-Petersburg, 2012) (in Rus.).

5. I. A. Nemirovskaya, A. P. Khaustov, M. M. Redina, Izvestiya Rossiiskoi Akademii Nauk. Seriya Geograficheskaya, 6, 49-56 (2018) (in Rus.).

6. A. P. Lisitsyn, Okeanologiya, 34(5), 735-747 (1994) (in Rus.).

7. A. P. Khaustov, and M. M. Redina., Geochemistry International, 55(1), 98-107 (2017).

8. M. B. Yunker, R. W. Macdonald, R. Vingarzan et al., Organic Geochemistry, 33, 489-515 (2002). 